Yi-Yun Cheng - University of Illinois at Urbana-Champaign,
yiyunyc2@illinois.edu
$\begin{aligned} & \text { Bertram Ludäscher } \\ & \text { ludaesch@illinois.edu }\end{aligned}-$ University of Illinois at Urbana-Champaign,

\title{
Exploring Geopolitical Realities through Taxonomies: The Case of Taiwan
}

\begin{abstract}
In the face of heterogeneous standards and large-scale datasets, it has become increasingly difficult to understand the underlying knowledge structures within complex information systems. These structures may encode latent assumptions that could be susceptible to issues such as ghettoization, bias, erasure, or omission. Inspired by a series of current events in the China-Taiwan conflict on the sovereignty of Taiwan, our research aims to develop methods that can elucidate multiple, often conflicting perspectives and hidden assumptions. We propose the use of a logicbased taxonomy alignment approach to first align and then reconcile distinct but overlapping taxonomies. We specifically examine three relevant taxonomies that list the world entities: (1) ISO 3166 for country codes and subdivisions; (2) the geographic regions of the US Department of Homeland Security; (3) the Center Intelligence Agency's World Fact Book. Our results highlight multiple alternate views (or Possible Worlds) for situating Taiwan relative to other neighboring entities. We hope that this work can be a first step to demonstrate how different geopolitical perspectives can be represented using multiple, interrelated taxonomies.
\end{abstract}

\section{Introduction}

In the face of heterogeneous standards and large-scale datasets, it has become increasingly important to understand the underlying knowledge structures within complex information systems. These structure may encode latent assumptions that could be susceptible to issues such as ghettoization, bias, erasure, or omission (Adler and Tennis 2013). Over the years, scholars in the field of Knowledge Organization (KO) have examined these issues in a wide range of research topics, such as in the classification of gender (Olson 2002), racial groups (Higgins 2016), and indigenous populations (Littletree and Metoyer 2015). They found that misrepresented library classifications tend to provide a one-sided perspective that favors a dominant world view (Adler 2016, Olson 2002).

Beyond the KO community, scholars from diverse disciplines have also taken interest in unveiling the presupposed structures in different contexts. In fact, the field of cartography and geographic information systems (GIS) have always been attentive to predetermined conformity behind the production of maps. Rundstrom (1995) has discussed the assimilation of western values on indigenous people's knowledge in the setting of GIS softwares and products. Soeller et al. (2016) have developed a tool to detect opaque personalization of online maps that showed different geopolitical country views based on locations. Stew- art et al.(2015) have developed a World Spatiotemporal analysis tool that incorporates a myriad of data sources and aims at representing world entities changes over time.

Inspired by a series of current events in the China-Taiwan conflict (Buckley and Horton 2019, Horton 2019) on the sovereignty of Taiwan, our research aims to provide a comprehensive and holistic view of multiple 'truths'. Similar to our prior work (Cheng et al. 2017, 
Cheng and Ludäscher 2018), we propose the use of a logic-based taxonomy alignment approach to reconcile distinct but overlapping taxonomies. We specifically examine three relevant taxonomies that enumerate countries in the world: (1) ISO 3166 country code; (2) the United States Homeland Security geographic regions; and (3) the Center Intelligence Agency's World Fact Book. We hope that this work can be a first step to demonstrate and reconcile different geopolitical realities through taxonomies.

\section{Related Work}

Embedded Structure and Knowledge Organization Systems

The impact of embedded beliefs within Knowledge Organization Systems (such as taxonomies, library classifications, thesaurus, ontologies, etc.) can be seen from two dimensions: misrepresentation of subjects, and social, cultural, or political influences.

Works by Hope Olson $(2002,2017)$ demonstrate a clear marginalization and systematic biases on the subjects in classification systems by examining cases on how females are often wrongly classified in racial groups and occupations in library classifications. Likewise, discussions on how racial representation in Dewey Decimal Classifications (DDC) (Higgins 2016), or how indigenous peoples knowledge and cultures are classified in major classifications (Green 2015, Hajibayova et al. 2016, Littletree and Metoyer 2015, Webster and Doyle 2008) have all reached similar conclusions that the unseen system biases are already entrenched in society (Mai 2016).

Moreover, classification is heavily influenced by social, cultural, or political matters. This was manifested in the apartheid South Africa in the 1950s as described in Bowker and Star (2000) who pointed that everyone needed to be classified into racial groups. Consequently, this classification system segregated where people can live, work, or even go to school.

Suggestions have been made to improve the status quo: for instance, Bowker and Star (2000) advocate to uncover the invisible infrastructures and retrace the original design intentions behind classifications, while Tennis (2012) proposes that the designer of classification should anticipate future changes and 'design for change'. While most of these studies aim to raise awareness of the entrenched, hidden structure within KOS from a critical theorist perspective, advocating changes can be difficult without empirical analysis or realistic actions.

\section{Geopolitical Realities}

Behind the creation of maps lies a number of social, political, and power assumptions that a nation, or a group of nations make about their boundaries and bordering territories. Geopolitical mapping is not simply mathematical or scientific reflection of the "real world", but rather used as "technologies of power" (Callahan 2009) that can shape a certain discourse about a nation. The subtle exhibitions of political messages on maps tell as much its social influence as power dynamics: omissions, or silences on maps only amplify the fact that elite groups exist and they are using maps as a means to 'promote an uneven dialogue' to the weak (Crampton 2001, Harley 1988, 1992). 
Extended empirical works confirm that maps, or modern days GIS systems are cultural and political products that overlook minority values, and advocate the geopolitical realities of those in power. Rundstrom (1995) have looked into the development of GIS tools and concludes that knowledge of the indigenous people may have been undermined due to the tacit social influences of the tool. Soeller et al. (2016) have developed MapWatch to detect Google maps when they provide personalized views of the world based on the location, especially at places where there are border conflicts (e.g. Russia, Ukraine and Crimea, etc.). They concluded that their close scrutiny to the hidden structure behind maps is not enough; a call for transparency on the border conflicts is very much more needed. Further, Stewart et al. (2015) have developed a World Spatiotemporal analysis project to aggregate all different data sources of world entities with an ontology-aided data model documenting entity name changes over time. Their intentions to integrate various data sources to disclose disparate features in these sources are also entailing a cellophane-like, permeable structure.

These empirical studies have examined controversial cases such as the Indo-Pakistan border conflict, South China Sea, or how the eastern Europe split from Yugoslavia to Croatia, Slovenia, and more (Soeller et al. 2016, Stewart et al. 2015), but empirical studies on other disputed regions such as Taiwan-China are yet to be discussed. Historically, Taiwan and China, have been in a long-standing dispute over sovereignties and there is yet to be a formal arrangement between the two geobodies. Recent decisions from a number of global companies' websites to drop the name "Taiwan" but keep only the capital city "Taipei" (Figure 1) as an indicator of the territory suggests there may be renegotiation of power between China and Taiwan (News 2018, Wee 2018). Moreover, most of the empirical studies mentioned above have explore these geopolitical issues at the actual map-level, a close inspection on how KO systems such as taxonomies can make a impact is not yet seen in these studies. This is an exemplar of prior events and has shown a pressing need for transparency in $\mathrm{KO}$ systems.

Figure 1. Major U.S. Airline web interface. A search on the keyword "Taiwan" would only return the cities Kaohsiung and Taipei; a search on China will return major cities in China but not Taipei; a search on other countries such as Vietnam will return "Hanoi, Vietnam" with both the city and country names included

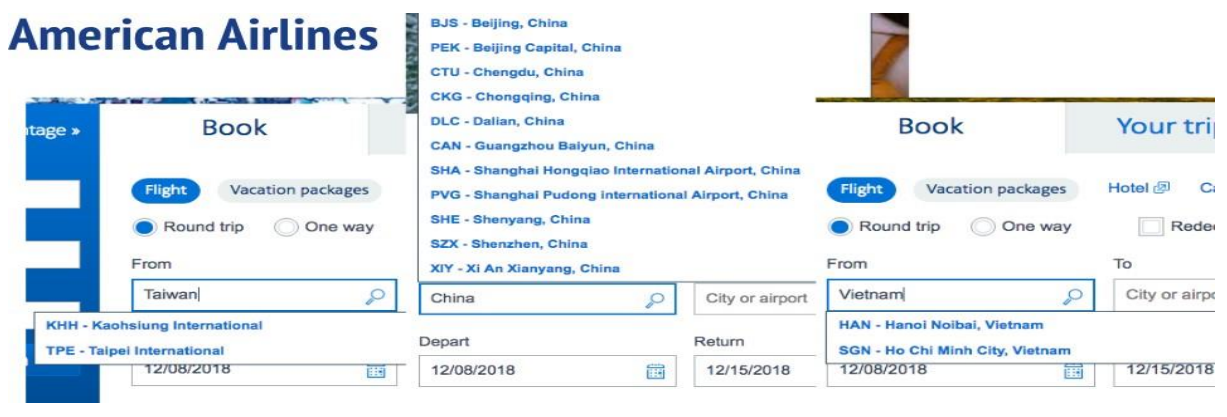


Yi-Yun Cheng and Bertram Ludäscher. 2019. Exploring Geopolitical Realities through Taxonomies: The Case of Taiwan. NASKO, Vol. 7. pp. 77-93.

\section{Method}

Data: The taxonomies

To demonstrate how logic-based taxonomy alignment can aid with reconciling conflicting boundary perspectives, we aim at aligning the vocabularies used for representing the world's entities, specifically zooming into the areas in Asia. Adopting the terminology used in Steward et al.(2015), we also use the term entities to avoid the disagreement among different data sources on what is a nation, country, or state.

Figure 2. ISO's presupposed hierarchical structure for the entities

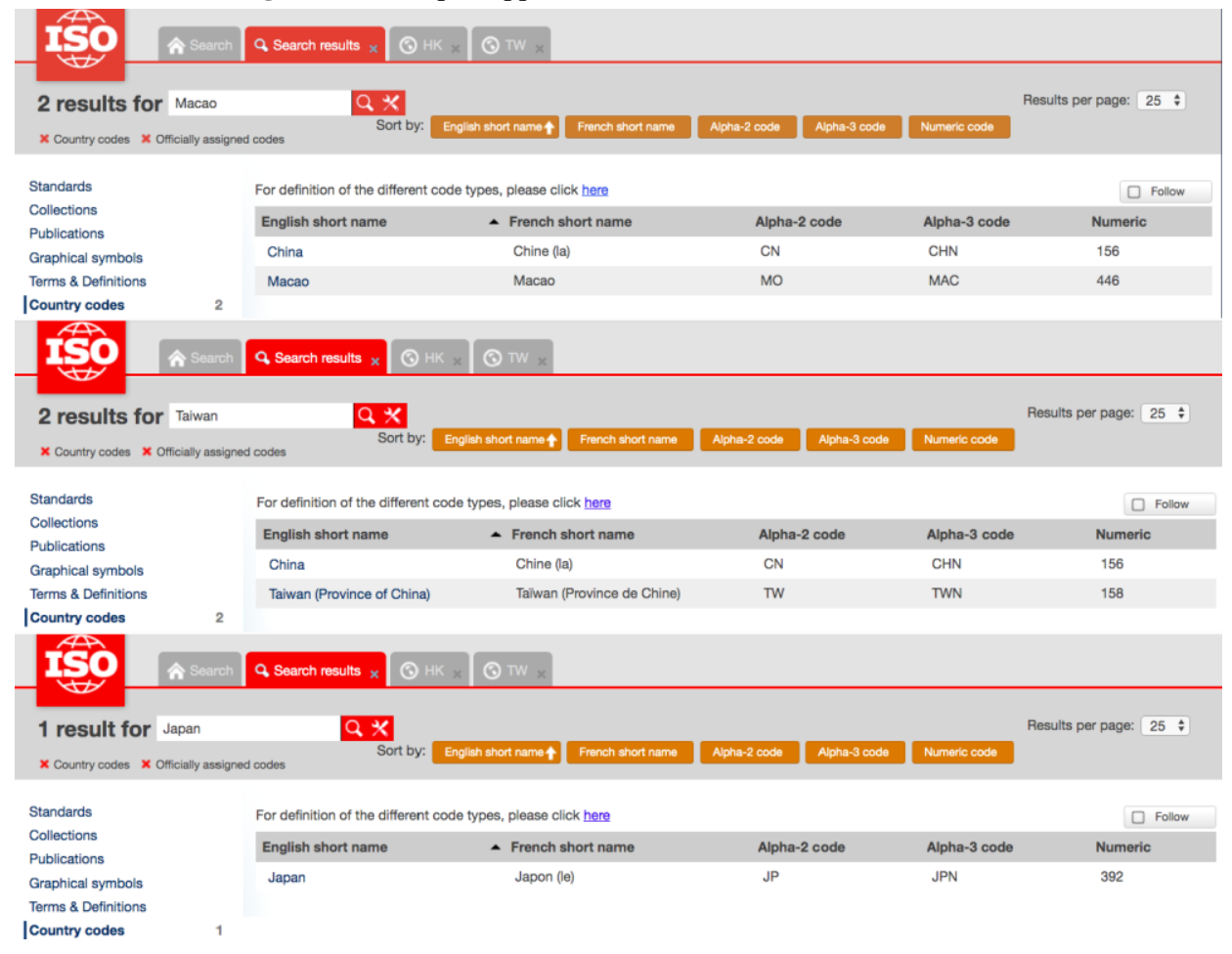

The three taxonomies we examined are:

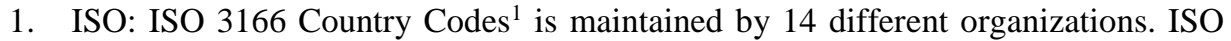
3166 reflects the United Nation's (UN) perspective of its member states. If an entity is not a UN member state, it will still be searchable on the ISO online platform, but it will not be listed as a country. Alternatively, it may be listed as a sub-entity of a

${ }^{1}$ 1ISO 3166: https://www.iso.org/iso-3166-country-codes.html 
particular country. For example, performing a search on the ISO platform on Macao (or Macau), Hong Kong, or Taiwan will result in two records for each, indicating that China is the parent of these entities; whereas performing a search on other entities such as Japan will only result in one record. Further, another layer of inconsistency is seen for the name shown for Taiwan. Unlike the naming for Macao being blatantly as Macao, ISO has to re-amplify that Taiwan is a "Province of China". See Figure 2 for ISO's implicit assumptions on sub-entities.

2. DHS: This is the taxonomy used in the United States Department of Homeland Security to identify the world's geographic regions ${ }^{2}$. The taxonomy itself is a flatter structure, meaning that there are no nested entities or sub-entities. Even for obvious cases where there might exist a parent country (e.g. Macau), DHS considers considered it as a disjoint entity from China. It is not explicitly stated whether this 'flat' structure means that each entity is its own independent state, or it merely means that the geographic area of these entities are disjoint from each other.

3. CIA World Fact Book: the Central Intelligence Agency World Fact Book ${ }^{3}$ consists of a comprehensive list, history, and facts of the world's entities. The World Fact Book also contains a hierarchy of entities. When certain entity is part of an independent state, that entity will contain one note on its Dependency Status ${ }^{4}$ and its Independence status will be listed as none. For example, Macau is noted as "special administrative region of the People's Republic of China" on its Dependency Status with the section on Independence noted as "none". As a counter example, Japan does not have an extra section on Dependency Status, and for its Independence section, "3 May 1947" is listed as the independence date. As for the case of Taiwan, there are neither indication of Dependency Status nor a section of Independence, though different Administrative divisions and National Holiday (from China, Hong Kong, and Macau) are described.

A comparison of the three taxonomies is shown in Table 1.

Table 1. A comparison of the three taxonomies examined.*Data are collected as of May, 2019.

\begin{tabular}{llcc}
\hline \multicolumn{4}{c}{ Maintained by } \\
\hline ISO 3166 & $\begin{array}{l}\text { 14 different organizations. } \\
\text { Usually reflecting UN } \\
\text { member states. }\end{array}$ & 5 & 250 \\
Department of & United States & 8 & 232 \\
CIA & United States & 11 & 267 \\
\hline
\end{tabular}

${ }^{2}$ DHS: https://www.dhs.gov/geographic-regions

${ }^{3}$ CIA Fact Book: https://www.cia.gov/library/publications/the-world-factbook/

${ }^{4}$ Dependency Status: "This entry describes the formal relationship between a particular nonindependent entity and an independent state." 
Logic Based Taxonomy Alignment Approach

Taxonomy. The definition of taxonomy within the scope of our work states that a taxonomy $\mathrm{T}$ is a hierarchical, tree structure of terms (or names) with each node having only one parent (with the exception of the root node having no parent). Each node is disjointing from its sibling node, meaning that two nodes on the same level in the tree are mutually exclusive of each other (sibling disjointness rule). The children in each branch of the tree are also considered to be the only children of the parent with no prodigal sons or long, lost siblings (parent coverage rule).

Taxonomy Alignment Problem (TAP). To compare two taxonomies T1 and T2, a set of articulations (relations) is used to specify how concept $\mathrm{X}$ in $\mathrm{T} 1$ relates to concept $\mathrm{Y}$ in $\mathrm{T} 2$. Here we use the five region connection calculus (RCC-5) to define the articulations: equals, overlaps, disjoint, includes, is_included_in. After we define how one taxonomy relates to another and input it in the Euler/X tool (an Answer Set Programming, Python based tool) or its latest update LeanEuler ${ }^{5}$, three different kinds of results will be presented. Euler/X will either conclude with (1) an inconsistent outcome with zero PW (n=0); (2) a single, uniquely merged PW T3 ( $=1)$; or (3) multiple merged PWs T3 (n 2), where each world is a possible reconciliation of how two taxonomies should be aligned. The best case scenario (2) with a unique world usually is the goal of a TAP where articulations are well-specified with no ambiguities. In cases of (1) and (3), the articulations between the two taxonomies T1 and T2 are incomplete or ambiguous,so the users may have to iteratively correct the articulations to reach the ultimate goal of a unique $\mathrm{PW}$.

Details of the Euler/X tool workflow and the steps for implementation are described in Cheng et al. (2017).

Interpretations. Recognizing that existing taxonomies or TAP may not have a ground truth to verify against, conscious human modeling decisions are often made in a TAP. For use cases that are of highly specialized knowledge, domain experts' insights are needed to specify how T1 relates to T2. Such cases can be found in works by Franz et al. $(2015,2016)$, in which the authors have explored the use of logic-based RCC-5 alignment in the field of biodiversity systematics with detailed expert's articulations.

In this research, we employed pairwise alignments with three taxonomies (ISO vs. DHS, ISO vs. CIA, and CIA vs. DHS). Two layers of interpretations are specified and explicitly explained here: (1) The taxonomies - we model each data source into a tree-like structure to the best of our knowledge or as reflected in the literature. This is demonstrated in the prior section describing the three taxonomies; (2) the articulations: given that the topic of our use case is intuitive, the articulations given between each concept in each pair of taxonomies are specified by us. For every use case, since the relation between Taiwan and China are undefined (and should not be defined by us), we do not specify any articulation between the two entities. Specifically, we left the nodes ISO.China, ISO.Taiwan, DHS.China, DHS.Taiwan, CIA.China, CIA.Taiwan unmapped from T1 to T2. Other regions in Asia are

${ }^{5}$ Euler X: https://github.com/EulerProject/EulerX; LeanEuler: https://github.com/idaks/LeanEuler 
Yi-Yun Cheng and Bertram Ludäscher. 2019. Exploring Geopolitical Realities through Taxonomies: The Case of Taiwan. NASKO, Vol. 7. pp. 77-93.

marked as equivalent in T1 and T2 if they share same or similar names (e.g. ISO. VietNam equals DHS. Vietnam). To mark all other entities in Asia as equivalent is a bold assumption, however, this is one way we believe can aid us in focus solely on the China-Taiwan entities. Further, the reason for leaving Taiwan and China blank is to address the latent structure within taxonomies as well as to keep an open stance of our perspectives.

Figure 3. Input data for ISO-DHS with all entities in Asia (Green boxes: ISO; yellow note boxes: DHS; purple dotted lines: the relations).

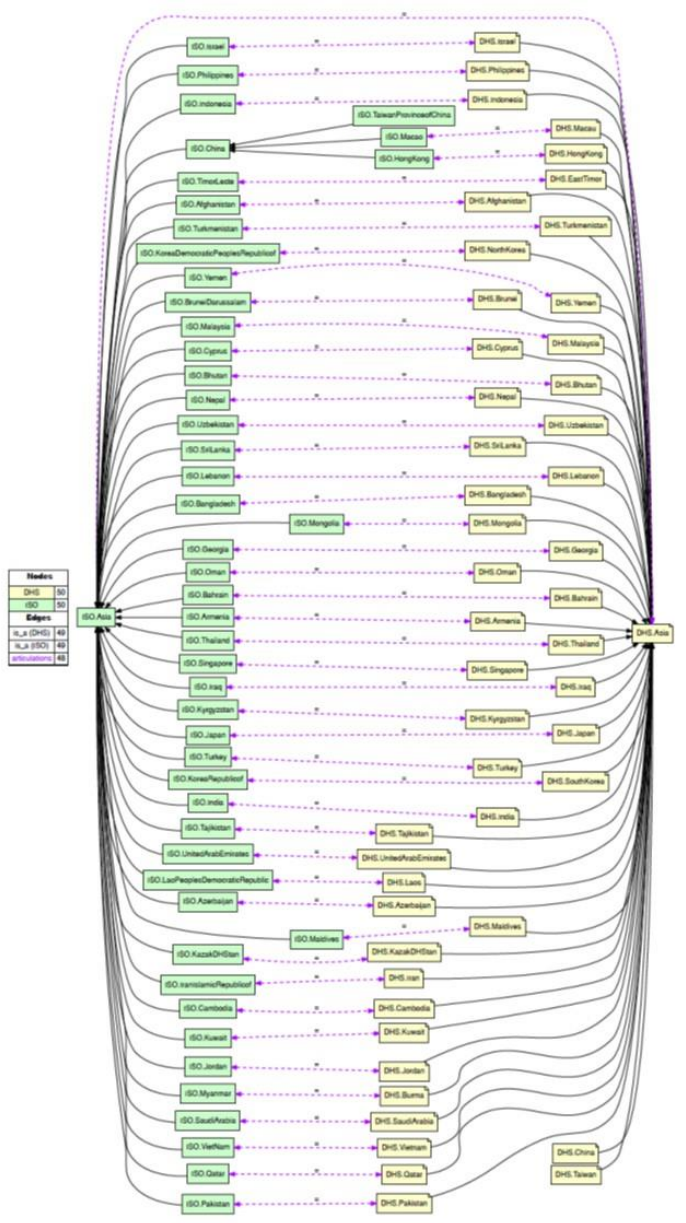




\section{Use Case 1: ISO-DHS Input Taxonomies}

For T1.ISO, because it has inconsistent naming and a presupposed hierarchical structure in which they give Taiwan the name "Taiwan (Province of China)" (see Figure 2), in this modeling situation, we then assume that China has four children: HongKong, Taiwan, Macao, and ChinaOther (to represent mainland China). As for T2.DHS, since it is a flat structure with every entity being its own independent state, we listed several entities in Asia and described them all as sibilings instead of chidren of a super-entity. Figure 3 is the visualization graph of the input alignment.

Since the full entities of Asia is too large, we also have an shortened version of entities. An abridged example of the Euler/X (or LeanEuler) input text file is as follows:

taxonomy ISO ISO3166

(Asia China VietNam AsiaOther)

(China HongKong Macao Taiwan ChinaOther)

taxonomy DHS HomelandSecurity

(Asia China ChinaOther HongKong Macau Taiwan Vietnam AsiaOther)

articulation ISO-DHS ISO-Homeland

[ISO.Asia equals DHS.Asia]

[ISO.HongKong equals DHS.HongKong]

[ISO.Macao equals DHS.Macau]

[ISO.VietNam equals DHS.Vietnam]

[ISO.ChinaOther equals DHS.ChinaOther]

[ISO.AsiaOther equals DHS.AsiaOther]

The shortened version of the input taxonomy can be visualized by Figure 4 .

Figure 4. Input data for ISO-DHS with abridged entities of Asia.

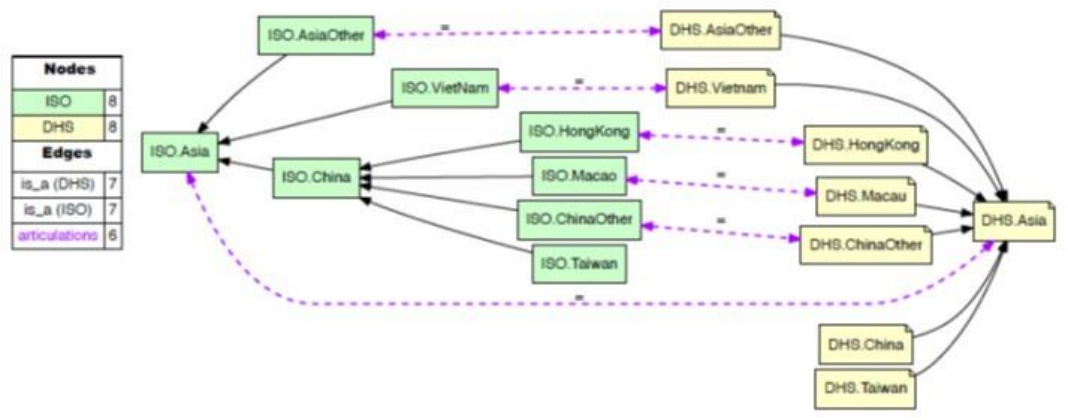


Yi-Yun Cheng and Bertram Ludäscher. 2019. Exploring Geopolitical Realities through Taxonomies: The Case of Taiwan. NASKO, Vol. 7. pp. 77-93.

Figure 5. One Possible World for with the full Asia lattice. Equivalent nodes (marked in grey round boxes) meant that those regions are reconciled and viewed as congruent; red arrows are the inferred relations from Euler/X.

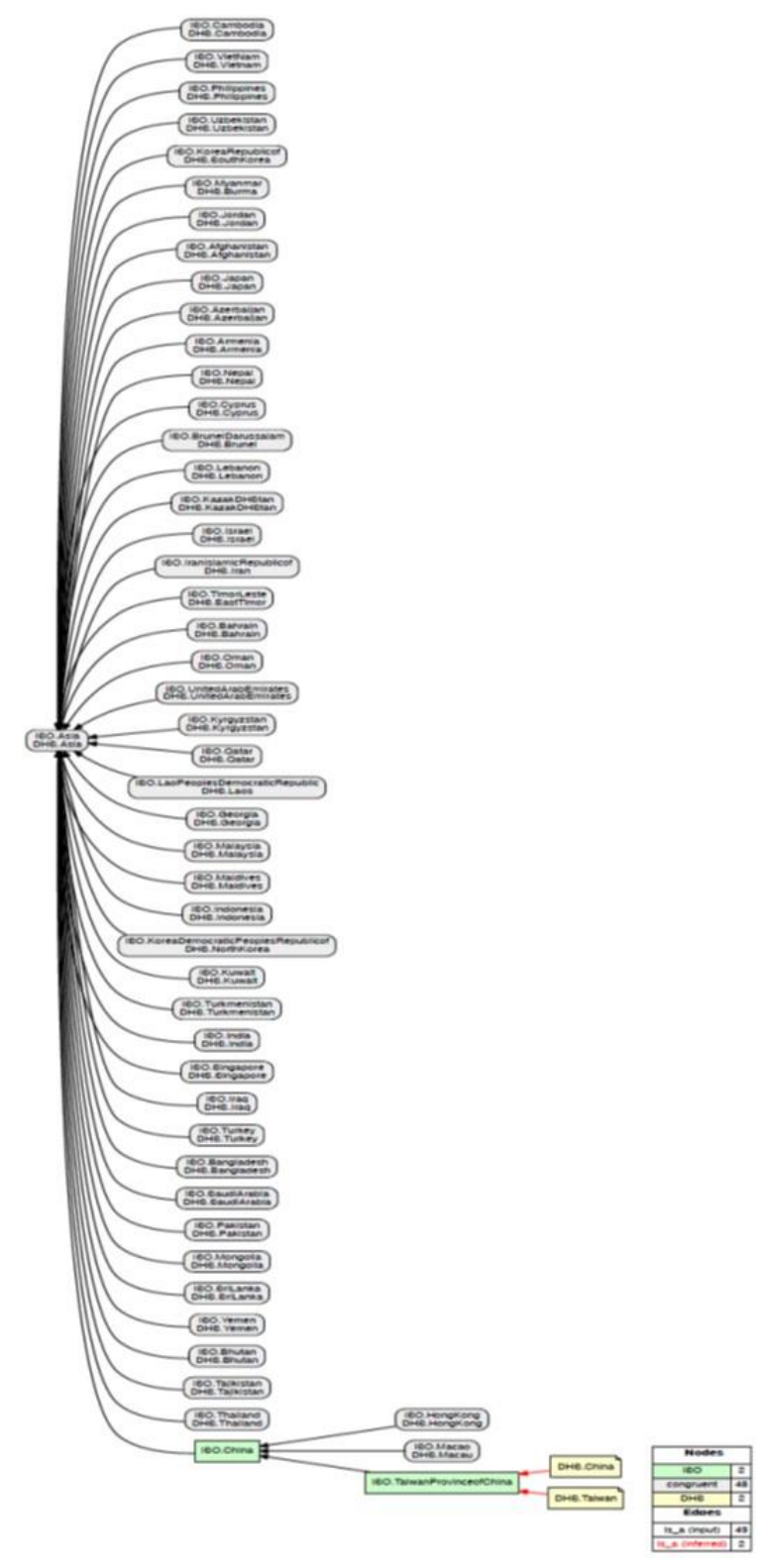




\section{ISO-DHS: merged T3 Possible World}

The TAP of ISO and DHS resulted in only one merged, unique Possible World. The full lattice of the Possible World can be seen in Figure 5. The merged possible world preserves the information from both taxonomy with extra information on how to make the TaiwanChina nodes consistent and logical in a unique world. In this world, both DHS.China and DHS.Taiwan are children of ISO.Taiwan, but ISO.Taiwan is a child of ISO.China (Figure 6). This means that there are parts (DHS.China and DHS.Taiwan) of the DHS taxonomy that should be viewed as children of the ISO taxonomy.

Figure 6. One Possible World with abridged entities of Asia. This created an interesting PW where one taxonomy T2.DHS has become sub-taxonomy of T1 when the nodes are not congruent (equivalent)(grey boxes).

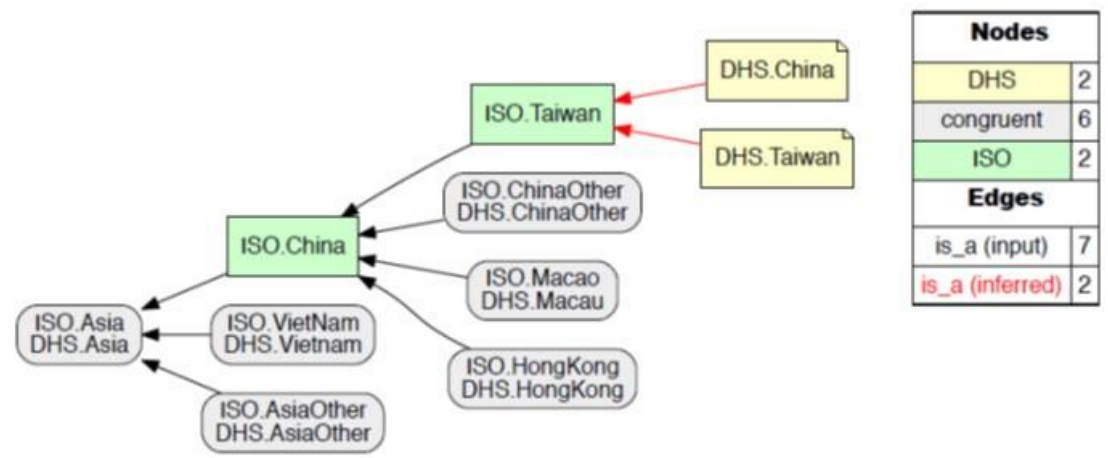

\section{Use Case 2: ISO-CIA}

Similar modeling situation has been devised for comparing ISO and CIA World Fact Book taxonomies. Aligning all the entities in Asia is sometimes illegible, therefore for the comparison from now on, we will focus only in parts of Asia with fewer entities.

\section{Input Taxonomies}

For use case 2 we have T1 for ISO, T2 for CIA Fact Book. Again, we have left the articulations for specifying either China or Taiwan blank, while marking the rest as equivalent. For this use case we pinpointed solely on Taiwan and China, and the other entities are all combined together to become one entity AsiaOther (Figure 7)). 
Figure 7. Input taxonomies for ISO-CIA.

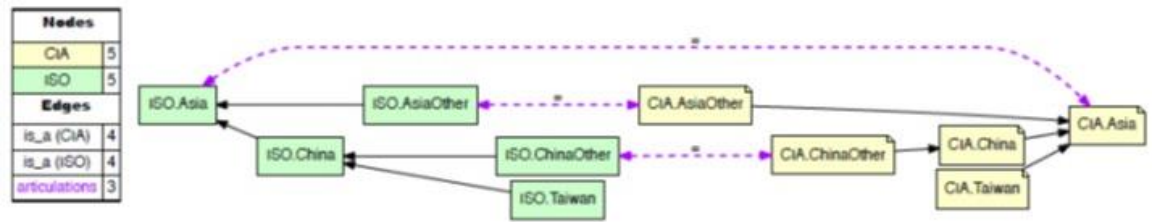

ISO-CIA: merged T3 Possible World

The merged Possible World looked as if they are a smaller subset of the result for the ISODHS alignment (Figure 6). However, because both ISO and CIA taxonomies have implicit hierarchical structures to specify entities, the merged T3 PW had subtle differences from the previous use case. CIA.China, CIA.ChinaOther and ISO.ChinaOther are considered the same; while ISO.Taiwan is equivalent to CIA.Taiwan. ISO.China is the super-entity of both CIA.China and CIA.Taiwan (or ISO.Taiwan). The two children are at equal level rather than one being superior than the other (See Figure 8).

Figure 8. Input data for ISO-CIA
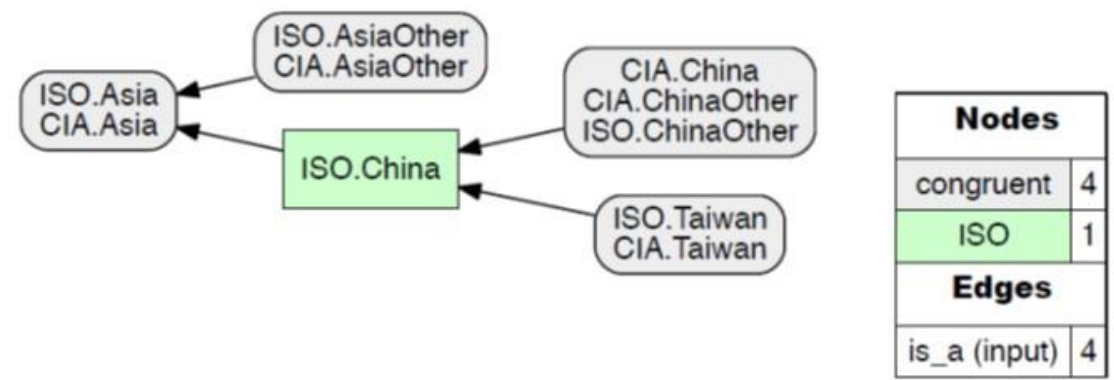

\section{Use Case 3: CIA-DHS Input taxonomies}

For use case 3, we compare the taxonomy T1.CIA with T2.DHS. We have once again focusing only on the two entities Taiwan and China, with all other entities combined as AsiaOther. Articulations between entities from T1 and T2 are marked as equivalent except for Taiwan and China. The interesting part about this use case is that the two taxonomies look identical in terms of structure, having a two-level structure with a root entity Asia and 
three children Taiwan, China, AsiaOther. Figure 9 shows the symmetry of the two taxonomies.

Figure 9. Input taxonomy for CIA-DHS

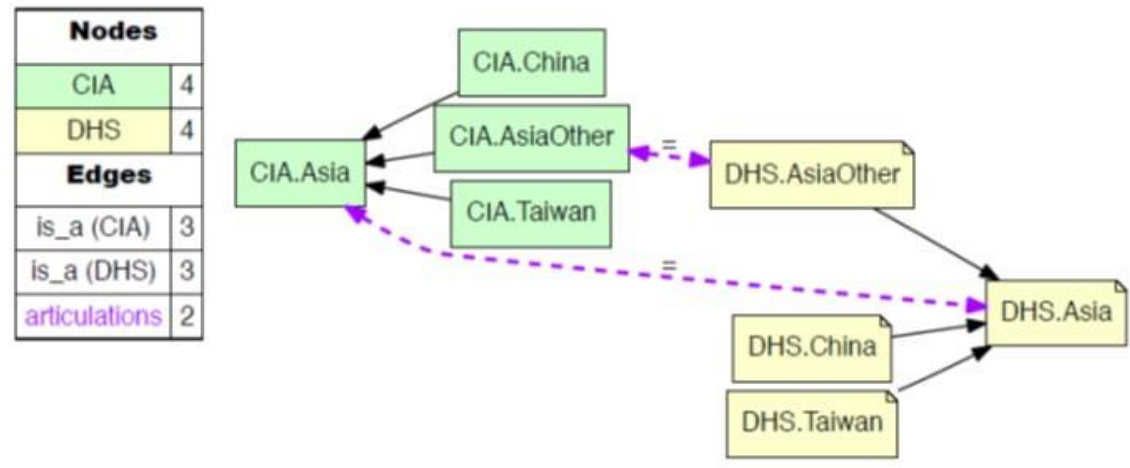

\section{CIA-DHS: merged T3 Possible Worlds}

This particular use cases presented different results than the previous use cases. Due to open, missing articulations among CIA.China, CIA.Taiwan, DHS.China, DHS.Taiwan, the results held room for ambiguities. This has resulted in seven possible worlds -7 different ways to merge the two taxonomies. Interestingly, these PWs may coincides with different social worlds that comprise different governmental (or political party's) perspectives (See Figure 10).

We can further examine the overlapping relations (red dotted lines) in Figure 10 and amplify that relations. Figure 11 shows this further dissection on how new regions (new entities) can be created in these 7 PWs. For example, in PW6, DHS.China can also be expressed by CIA.China minus the entity DHS.Taiwan; similar expressions can be said for CIA.Taiwan. Further, there exists a new entity CIA.China intersects DHS.Taiwan that is included in both CIA.China and DHS. Taiwan (Pink box in PW6 of Figure 11). More complex situations are seen in PW3 with four new entities, each has two parent nodes. To make sense of these new entities, for instance, the pink node in the bottom have three different ways to express the same entity: "CIA $\leftarrow$ DHS.Taiwan" OR "CIA.Taiwan\ DHS.China" OR "DHS.Taiwan\CIA.China". 
Yi-Yun Cheng and Bertram Ludäscher. 2019. Exploring Geopolitical Realities through Taxonomies: The Case of Taiwan. NASKO, Vol. 7. pp. 77-93.

Figure 10. 7 possible worlds possibly representing different perspectives on the China-Taiwan relationship. Based on our background knowledge and conjectures, it roughly corresponds to: PW1

may be how the rest of the world sees T-C relationship - they might not even be aware of the conflicts; PW2 may be how the Democratic Progressive Party (DPP) views the T-C relationship Taiwan and China are separate countries; PW3 may be how neighboring governments view the T-C relationship - the two regions overlaps in multiple ways and it is difficult to state what is true (red dotted lines means overlaps); PW4 may correspond to political extremists's view in Taiwan; PW5 and PW6 may be how the Kumintang (KMT) party view the situation - Taiwan has its own individual identity but still overlap partially with China; PW7 may correspond to how the China government view this relationship - Taiwan is part of China.

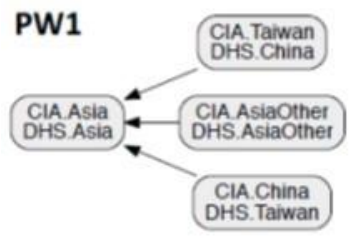

PW4
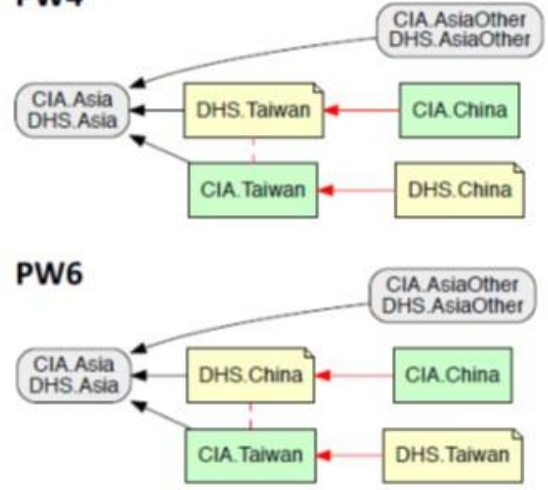

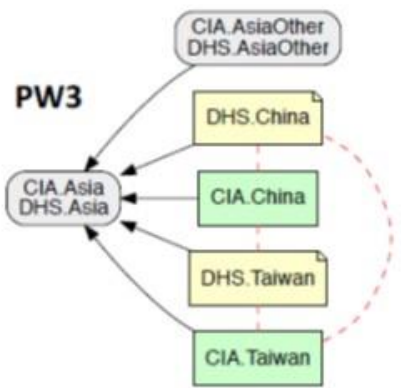

PW5

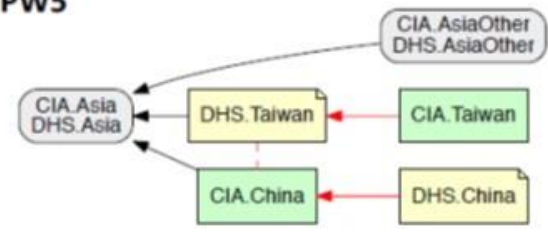

PW7

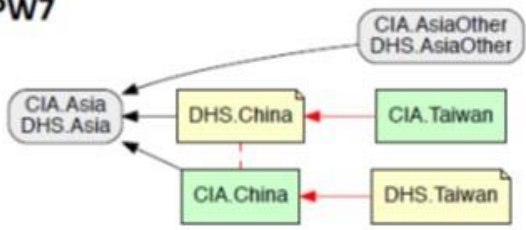


Yi-Yun Cheng and Bertram Ludäscher. 2019. Exploring Geopolitical Realities through Taxonomies: The Case of Taiwan. NASKO, Vol. 7. pp. 77-93.

Figure 11. Zooming in further to examine the overlapping relations (red dotted lines) of the 7 PWs. Pink boxes represent new names for the entities; The asterisk sign means intersection of two entities; the slash sign means minus.
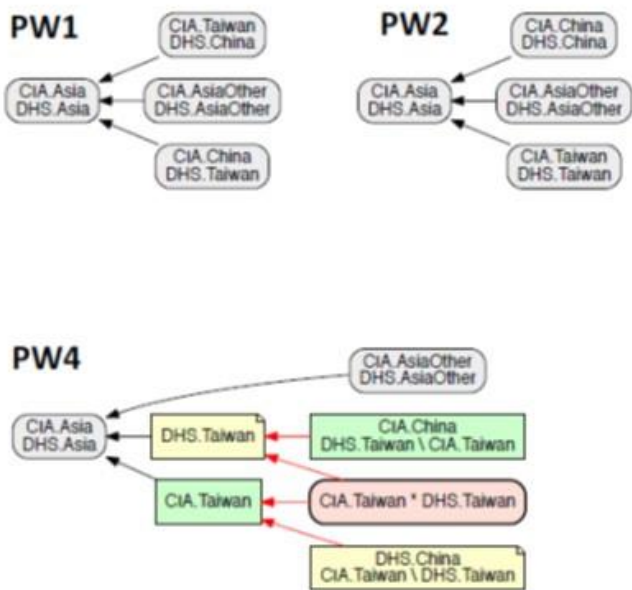

PW6

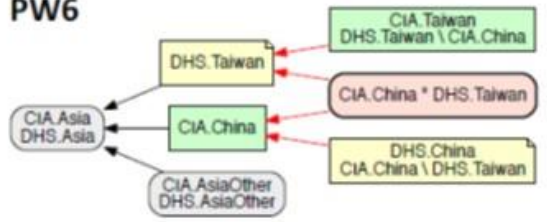

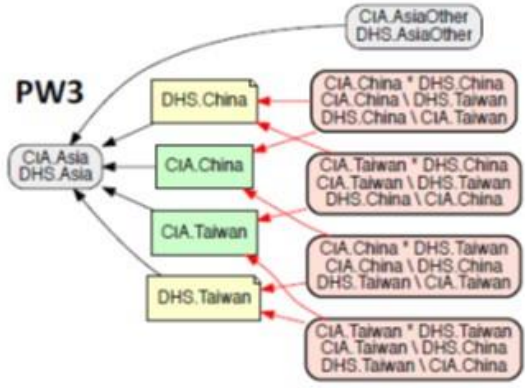

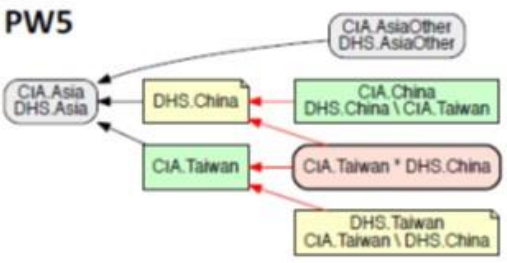

PW7

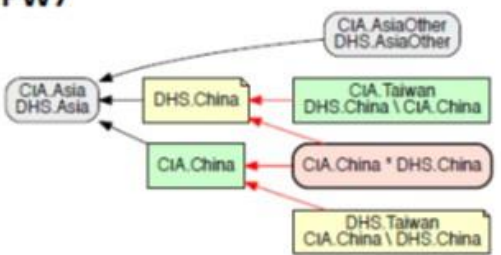

These newly created entities (pink boxes) might look complicated, but it is informative to specify which part is truly overlapping with which other entities. Moreover, this also shows that in every PW, the modeling situations for Taiwan and China perhaps can not be as incisive given the historical ties and entangled relationships. All the artifacts, including the input taxonomies as well as the output visualizations, used and produced by this study can be freely accessed on our Github Repository: https:/github.com/EulerProject/NASKO19 .

\section{Discussion and Conclusion}

This paper is our first attempt at looking into the disputed geographic regions and sovereignties in the world, focusing on the case of Taiwan. The three taxonomies chosen in this study serve different purposes: the ISO standard (ISO) conforms with the United Nation's viewpoints of the world with a more reserved stance on its member stances; the U.S. Homeland Security (DHS) taxonomy provides a more liberal, flat worldview; whereas the 
CIA World Fact Book (CIA) strikes a balance of the former two taxonomies. We have employed a logic-based, taxonomy alignment approach to align three use cases - ISO vs. DHS, ISO vs. CIA, and DHS vs CIA. The results of these three use cases provided different modeling situations for the two entities (Taiwan and China) and our taxonomy alignment results (specifically the use case on DHS vs. CIA) interestingly coincides with different perspectives in different social worlds. The merged Possible Worlds T3 in these use cases may be viewed as a new taxonomy that integrated multiple sources, and the PWs T3 may then later become a new input of a new taxonomy alignment problem.

Latent assumptions within information systems is not unusual. Despite that there are multitudinous taxonomies about the world's entities, a copious existing information systems endorse the ISO 3166 country codes. For instance, the Library of Congress (LOC) 6 adopts the ISO country code for both the MARC 21 standard 7 and the LOC classifications 8 . The MARC 21 code list makes subtle distinction between China and China (Republic: 1949- ), where the former is specifically pointing to the mainland, the latter is meant for Taiwan. On the other hand, the LOC classification conforms entirely to the ISO standard and lists Taiwan as a sub-division of China. The reason LOC adopts the ISO country code is not explicitly manifested, and how such choice may lead to further unexpected, unforeseen actions or consequences in information systems is out of the scope of this research. However, through the lens of taxonomies and taxonomy alignment, it is exemplified that geopolitical realities exist in knowledge structures and we believe making these assumptions and choices explicit is crucial in this information age. Moreover, to raise awareness that there are hidden structures within a KOS is only the first step, to present a more holistic view on the possible worlds of these geopolitical realities simultaneously will be inevitable.

Whether Taiwan is an independent nation or part of China still remains a moot point. This research is subjected to the limitation of researcher's bias, because Taiwan is the first author's homeland. In spite of the emotional, close ties the researchers might have with Taiwan, we have tried to limit any personal thoughts on the Taiwan and China relation. Yet, we have attempted with even more efforts on making any interpretations or modeling situations of the use cases unequivocal in order to keep a open stance and embrace different opinions on this issue.

Future work on looking at geopolitical realities in taxonomies or other Knowledge Organization Systems will attempt to incorporate more data sources, align other entities in the world, and discuss perceived boundaries between minority groups and the authorities (e.g. indigenous people versus the government). We believe this line of work can be of great contributions to demonstrate different geopolitical realities in taxonomies and KOS as well as to give voices to the silenced values.

\section{References}

M. Adler. 2016. The case for taxonomic reparations. Knowledge Organization 43(8) (2016): 630-640. M. Adler and J. Tennis. 2013. Toward a taxonomy of harm in knowledge organization systems. Knowledge Organization 40(4): (2013).

G.C. Bowker and S.L. Star. 2000. Sorting things out: Classification and its consequences. MIT press. 
Yi-Yun Cheng and Bertram Ludäscher. 2019. Exploring Geopolitical Realities through Taxonomies: The Case of Taiwan. NASKO, Vol. 7. pp. 77-93.

C. Buckley and C. Horton. 2019. Xi Jingping warns Taiwan that unification is the goal and force is an option. https://www.nytimes.com/2019/01/01/world/asia/xi-jinping-taiwan-china.html.

W.A. Callahan. 2009. The cartography of national humiliation and the emergence of China's geobody. Public Culture 21(1) (2009): 141-173.

Y. Cheng, N. Franz, J. Schneider, S. Yu, T. Rodenhausen, and B. Ludäscher. 2017. Agreeing to disagree: Reconciling conflicting taxonomic views using a logic-based approach. Proceedings of the Association for Information Science and Technology 54(1) (2017): 46-56.

Y. Cheng and B. Ludäscher. 2018. Full of beans: a study on the alignment of two flowering plants classification systems. Proceedings of the 18th European Networked Knowledge Organization Systems Workshop (NKOS) (2018).

J.W. Crampton. 2001. Maps as social constructions: power, communication and visualization. Progress in human Geography 25(2) (2001): 235-252.

N.M. Franz, M. Chen, S. Yu, P. Kianmajd, S. Bowers, and B. Ludäscher. 2015. Reasoning over taxonomic change: exploring alignments for the Perelleschus use case. PloS one 10(2) (2015): e0118247.

N.M. Franz, N.M. Pier, D. M. Reeder, M. Chen, S.Yu, P.Kianmajd, S. Bowers, and B. Ludäscher. 2016. Two influential primate classifications logically aligned. Systematic biology 65(4) (2016): 561-582.

R. Green. 2015. Indigenous Peoples in the US, Sovereign Nations, and the DDC. KNOWLEDGE ORGANIZATION 42(4) (2015): 211-221.

L. Hajibayova, W. Buente, L. Quiroga, and S. Valeho-Novikoff. 2016. Representation of Kanaka Maoli (Hawaiian) culture: A case of hula dance. In Proceedings of the 79th ASIS\&T Annual Meeting: Creating Knowledge, Enhancing Lives through Information \& Technology. American Society for Information Science, 128.

J.B. Harley. 1988. Silences and secrecy: the hidden agenda of cartography in early modern Europe. Imago mundi 40(1) (1988): 57-76.

J.B. Harley. 1992. Rereading the maps of the Columbian encounter. Annals of the Association of American Geographers 82(3) (1992): 522-542.

M. Higgins. 2016. Totally Invisible: Asian American Representation in the Dewey Decimal Classification, 1876-1996. KNOWLEDGE ORGANIZATION 43(8) (2016): 609-621.

C. Horton. 2019. Taiwan's President, defying Xi Jinping, calls unification offer 'impossible'. https://www.nytimes.com/2019/01/05/world/asia/taiwan-xi-jinping-tsai-ing-wen.html.

S. Littletree and C.A. Metoyer. 2015. Knowledge organization from an indigenous perspective: The Mashantucket Pequot thesaurus of American Indian terminology project. Cataloging \& Classification Quarterly 53(5-6) (2015): 640-657.

J. Mai. 2016. Marginalization and exclusion: Unraveling systemic bias in classification. KNOWLEDGE ORGANIZATION 43(5) (2016): 324-330.

Bloomberg News. 2018. U.S. Airlines alter websites to meet China's demand on Taiwan's name. https://www.bloomberg.com/news/articles/2018-07-25/u-s-airlines-alter-websites-to-meet-chinademand-on-taiwan-name.

H.A. Olson. 2002. The power to name: locating the limits of subject representation in libraries. Springer Science \& Business Media.

H.A. Olson. 2017. Social influences on classification. In Encyclopedia of library and information sciences. CRC Press, 4204-4211.

R.A. Rundstrom. 1995. GIS, indigenous peoples, and epistemological diversity. Cartography and geographic information systems 22(1) (1995): 45-57. 
Yi-Yun Cheng and Bertram Ludäscher. 2019. Exploring Geopolitical Realities through Taxonomies: The Case of Taiwan. NASKO, Vol. 7. pp. 77-93.

G. Soeller, K. Karahalios, C. Sandvig, and C. Wilson. 2016. Mapwatch: Detecting and monitoring international border personalization on online maps. In Proceedings of the 25th International Conference on World Wide Web. International World Wide Web Conferences Steering Committee, 867-878.

R.N. Stewart, J.O. Piburn, A. Sorokine, A.T. Myers, and D.A. White. 2015. World Spatiotemporal Analytics and Mapping Project (WSTAMP): Discovering, Exploring, and Mapping Spatiotem- poral Patterns across the World s Largest Open Source Geographic Data Sets. Technical Report. Oak Ridge National Lab.(ORNL), Oak Ridge, TN (United States).

J.T. Tennis. 2012. The strange case of eugenics: A subject's ontogeny in a long-lived classification scheme and the question of collocative integrity. Journal of the American Society for Information Science and Technology 63(7) (2012): 1350-1359.

K. Webster and A. Doyle. 2008. Don't class me in antiquities! Giving voice to Native American materials. Radical cataloging: Essays at the front (2008): 189-197.

S. Wee. 2018. Giving In to China, U.S. Airlines Drop Taiwan (in Name at Least). https://www.nytimes. com/2018/07/25/business/taiwan-american-airlines-china.html. 